University of Louisville

ThinkIR: The University of Louisville's Institutional Repository

Faculty Scholarship

2012

\title{
Multisite recruitment and data collection among older adults : exploring methods to conserve human and financial resources.
}

Valerie Lander McCarthy

University of Louisville, valerie.mccarthy@louisville.edu

Karen Cassidy

University of Louisville

Follow this and additional works at: https://ir.library.louisville.edu/faculty

Part of the Geriatric Nursing Commons, and the Gerontology Commons

Original Publication Information

This is a pre-copy-editing, author-produced PDF of an article accepted for publication in the Journal of Nursing Measurement, volume 20, issue 2, in 2012, following peer review. The definitive publisherauthenticated version is available online at DOI: 10.1891/1061-3749.20.2.142

This Article is brought to you for free and open access by ThinkIR: The University of Louisville's Institutional Repository. It has been accepted for inclusion in Faculty Scholarship by an authorized administrator of ThinkIR: The University of Louisville's Institutional Repository. For more information, please contact thinkir@louisville.edu. 


\author{
Multisite Recruitment and Data Collection Among Older Adults: Exploring Methods to \\ Conserve Human and Financial Resources \\ Valerie Lander McCarthy, PhD, RN, \& Karen Cassidy, EdD, MSN, APRN \\ University of Louisville, Kentucky
}

(2012). Journal of Nursing Measurement, 20(2), 142-152.

Doi: http://dx.doi.org/10.1891/1061-3749.20.2.142

Corresponding author:

Valerie Lander McCarthy, PhD, RN

Associate Professor

School of Nursing

University of Louisville

Louisville, KY 40292

vemcca01@1ouisville.edu

502-852-8395 


\begin{abstract}
The purpose of this article is to describe strategies that were effective in recruitment and data collection among older adults in three quantitative studies while decreasing costs in terms of time and money. Factors effective in reducing use of investigators' time and expenses included limiting exclusion of data because of abnormal Mini-Cog scores by careful initial screening and avoiding repeated reminders or follow-up, collecting data in small groups, collapsing consent, dementia screening, and data collection into single sessions, as well as accommodating for sensory and literacy deficits. The cross-sectional, descriptive studies were conducted among community-dwelling older adults attending senior citizen centers and among older adults in independent or assisted living apartments within continuing care retirement communities (CCRCs). In the latest study, a convenience sample $(N=152)$ was recruited and data collection was completed in 4 weeks at a total cost of less than $\$ 5,000$. Methods common to qualitative research and those commonly used in community-based research were adapted to reduce time and costs for recruitment, screening, and data collection. Given limited availability of research funding, other nursing researchers may find one or more of these methods useful.
\end{abstract}

Keywords: recruitment; data collection; older adults; small groups; innovation 


\section{Multisite Recruitment and Data Collection Among Older Adults: Exploring Methods to Conserve Human and Financial Resources}

Recruiting representative samples of older adults for research studies can be challenging (Boles, Getchell, Feldman, McBride, \& Hart, 2000; Ory et al., 2002; Sullivan-Bolyai et al., 2011), especially considering the wide range of cognitive and functional abilities among the older adult population (New England States Consortium [NESC] Proceedings, 2000). Recruitment also is a costly process (Blackman, Kamimoto, \& Smith, 1999; Ory et al., 2002). As an example, in a study of health promotion among older adults of 746 individuals screened in a baseline visit, only 156 subjects were eligible. Recruitment costs were reported as $\$ 2,750$ for television advertising, $\$ 2,369$ for newspaper advertising, $\$ 5,691$ for radio ads, and $\$ 3,931$ for postal mailings (McDermott et al., 2009). Even just staff time and incidental expenses in a multisite study examining effective strategies for recruitment of older adults was estimated at $\$ 300$ per participant (Ory et al., 2002), whereas another study found these costs totaled $\$ 439$ per participant (Katula et al., 2007).

Recruiting older adult subjects at the time of hospital discharge or during a visit to a physician's office or clinic may be complicated by immediate health concerns that take priority over participating in a research study and may influence responses for those who do choose to participate (Woodall, Morgan, Sloan, \& Howard, 2010). Additionally, significant numbers of older adults fear being victimized by fraud and are reluctant to respond to requests to participate in research studies. This is particularly true among minority and low-income older adults (Meiner \& Lueckenotte, 2005).

Recruitment is generally a two-step process that begins with recruiting potential participants, followed by initial screening to determine if inclusion criteria are met before 
obtaining consent and collecting data. Screening for inclusion criteria is complicated among older adults by the prevalence of dementia in this population. U.S. federal regulations for the conduct of research involving human subjects provide specific guidance when dealing with a population with compromised capacity to consent, such as those with mild dementia (U. S. Department of Health and Human Services [DHHS], 2009). According to the U. S. DHHS, "Impaired decision-making capacity need not prevent participation in research, but additional scrutiny and safeguards are warranted for research involving individuals with such impairments" (U.S. DHHS, 2009, Introduction, para. 1). This additional scrutiny is provided by accredited institutional review boards which are responsible for interpreting federal regulations and approving and monitoring study protocols to ensure compliance with relevant federal regulations.

Data collection among older adults also offers unique challenges. Survey data collection methods commonly include mail, telephone, and in-person modalities. Previous studies have indicated that older adults' survey responses may be affected by cognitive differences between younger and older (65 years and older) populations as well as by differences in stamina, education levels, and familiarity with tasks such as answering survey questions (NESC, 2000). Collecting data via mail or telephone surveys also may be affected by fatigue, visual impairments, hearing loss, and apprehension about fraudulent and deceptive practices (Knäuper, Schwarz, Park, \& Fritsch, 2007; Parker \& Dewey, 2000). It has been suggested that low literacy levels among older adults may also be a factor, particularly among minority or disadvantaged groups (Centers for Disease Control and Prevention [CDC], 2009). Thus one-on-one interviews are recommended instead of mail or telephone surveys; length of surveys should be minimized to 
avoid fatigue and accommodations for sensory or literacy limitations should be considered (NESC, 2000).

\section{DESCRIPTION OF THREE CROSS-SECTIONAL SURVEY STUDIES}

A series of three cross-sectional survey studies used standardized questionnaires, administered to small groups of older adults $(n=5-8)$ during single data collection sessions encompassing consent, dementia screening, and completion of questionnaires while accommodating for sensory and literacy limitations. Each study was approved by a fully accredited university internal review board and complied with federal regulations for human subjects' protection. Inclusion criteria for all three studies were older adults aged 65 years or older, physical and sensory ability to complete the survey process (with accommodation if needed), and cognitive ability to understand survey procedures indicated by normal scores on the Mini-Cog dementia test (Borson, Scanlan, Brush, Vitaliano, \& Dokmak, 2000).

The Mini-Cog is a 3-min recall and clock-drawing test used to screen for dementia with results comparable to the Mini-Mental State Exam (Folstein, Folstein, \& McHugh, 1975). Initial screening protocols included scripted explanations of inclusion criteria and dementia screening; participants were told that data analysis was limited to data from participants with normal dementia test scores. Subsequently, after review and explanation of inclusion criteria, written consent, dementia screening, and data collection were completed in the small group setting. The protocols were specifically designed to protect the dignity and privacy of participants with abnormal Mini-Cog scores by allowing them to complete the questionnaires regardless of test scores.

The three studies reported here, in the order in which they were conducted, include (a) a 
pilot study using a convenience sample $(N=20)$ recruited at a moderate-income continuing care retirement community (CCRC); (b) a larger study using a random sample recruited in a lowincome CCRC $(N=123)$; and (c) a follow-up study using a convenience sample recruited at multiple sites $(N=152)$ in both a CCRC and among community-dwelling older adults. Figure 1 provides a model of the protocols used in all three studies.

--- FIGURE 1 HERE ---

The pilot study (McCarthy, 2009a) used a convenience sample of older adults living in independent or assisted-living apartments in a moderate-income CCRC. The study was designed to examine methods for recruitment, dementia screening, and data collection and to identify instruments for future use. The pilot study confirmed the feasibility of (a) using Mini-Cog scores to screen for dementia at a level that would be likely to interfere with the survey process; (b) limiting exclusion of data because of abnormal Mini-Cog scores by careful initial screening and avoiding repeated reminders or follow-up; (c) collecting data in small groups ( $n=5$ to 8 ); (d) collapsing consent, dementia screening, and data collection into single 1-1.5 hr sessions; and (e) accommodating for sensory and literacy deficits. Surprisingly, given the small sample size, findings also included a statistically significant model which supported the primary hypothesis of the second study.

The second study (McCarthy, 2009b, 2011) was conducted a year later among a stratified random sample $(N=123)$ of non-demented older adults living in independent or assisted living apartments in a low-income CCRC. Recruitment methods included flyers, informational presentations at resident meetings, and rapport building with CCRC staff who provided encouragement for residents to participate in the study if invited. Following residents' approval 
for the principal investigator (PI) to make phone contact, a sampling frame was used to telephone randomly selected residents for initial screening.

The PI, an experienced gerontological nurse, performed the initial telephone screening, giving a detailed explanation of the study protocol. Explanation of the protocol included that dementia screening would be done after written consent and before completing questionnaires and that normal scores were required for data to be included in the study. Questions were asked to explore each potential participant's understanding of the protocol and willingness to participate. Participants who evidenced cognitive deficits in the telephone screening were not scheduled for data collection. Eligible participants who agreed to participate were scheduled for data collection sessions at a time of their choice and a single written reminder was placed in the participant's mailbox.

Data collection was conducted in small groups ( $n=5$ to 8$)$ in a private meeting room at the CCRC. Participants received binders containing the consent form, Mini-Cog test, and all questionnaires, and the protocol and written consent form were reviewed and signed. Exclusion of data if dementia screening was abnormal was discussed. Scripted instructions were given for completing written informed consent, the Mini-Cog test, and questionnaires. Accommodations were made for sensory or literacy deficits, including using 14-point font size, reading all items aloud at least twice, and providing laminated cards in 24-point font size with scales for each questionnaire. At the end of the session, participants were thanked, asked to refrain from sharing survey items with other potential participants, and received $\$ 5$ gift cards to a nearby market as a gesture of appreciation. Findings included a significant regression model supporting the study's hypotheses. 
The third study $(N=152), 18$ months after the second study, was intended to extend the findings of the second study to a broader, more representative sample. Using most of the same recruitment and data collection methods used in the second study, a convenience sample for the third study was recruited at three sites: (a) an upper-income CCRC $(n=52)$ and among community-dwelling older adults at two senior citizen centers, (b) one in a low-income neighborhood $(n=49)$, and (c) the other in a moderate-income neighborhood $(n=51)$. In addition to the recruitment methods used in the previous randomized study, staff at each of the three sites actively encouraged potential participants to contact the PI for information about enrolling, and participants were asked to suggest to their peers that they also contact the PI for information. The protocol for screening, scheduling data collection sessions, written consent, dementia testing, data collection, and accommodation for sensory and literacy deficits remained the same with the exception that community dwelling participants recruited at the senior citizen centers did not receive written reminders of the scheduled data collection sessions. Participants at the CCRC received $\$ 10$ gift cards to a nearby market, whereas those at the senior citizen centers were included in a drawing for $\$ 50$ cash prizes. Again, a significant regression model supporting the study's main hypothesis was found.

\section{ADAPTED METHODS FROM QUALITATIVE AND COMMUNITY-BASED RESEARCH}

In general, methods common to qualitative research and those used in community-based research were adapted to meet the need for standardization and rigor in quantitative survey studies. For instance, in qualitative research, interaction between researcher and subjects to establish trust and build relationships is a common goal, whereas in quantitative studies, investigators maintain a distance to avoid influencing participants' responses. In the studies described here, it was important to gain participants' trust and relieve concerns about sharing 
information with strangers. At the same time, scientific rigor was maintained by strictly adhering to a standardized protocol to preserve independence of scores and participant privacy.

Collaboration between researchers and staff at each site was crucial for individualizing effective recruitment methods to the specific site. Although site selection was based on racial, ethnic, and socioeconomic characteristics to assure a diverse sample, the level of interest and cooperation among staff was also considered. Partnership with the administration and staff was essential in identifying usual routines and social activity schedules, so as to schedule data collection sessions at convenient times. Establishing a trusting relationship with administration and staff was beneficial to recruitment efforts because these partners were willing to offer reassurance and encouragement to potential participants who frequently fear being taken advantage of by strangers.

Developing rapport and interacting with participants to create a pleasant, social atmosphere while using a strict standardized protocol were both important to enable data collection to be done in small groups instead of using one-on-one interviews and to increase the likelihood of snowball sampling (i.e., encouraging peers to enroll in the study). Strict use of a standardized protocol while carefully explaining the reasons for restrictions and the rigid steps to the process was well accepted by participants when a brief discussion of the rationale was provided. Without exception, participants abided by instructions to remain silent and to avoid asking for items to be explained or clarified.

Ethical concerns were addressed in deciding to collapse written consent, dementia screening, and data collection into single sessions. In compliance with federal regulations protecting human subjects (U.S. DHHS, 2009) and the rules of the university institutional review board, it was deemed important that written consent be obtained prior to administering the 
Mini-Cog test. Thus informed consent, dementia screening, and completion of questionnaires were conducted in a single session. Mini-Cog scores were not known to researchers until after data collection was completed. Data was later excluded from analysis if the Mini-Cog test was abnormal. During data collection sessions, even when dementia-type behaviors were observed, all participants were allowed to complete the set of questionnaires to preserve dignity and privacy. Abnormal Mini-Cog results were reported to professional staff of facilities to ensure participants' safety; permission for this was also included in the consent form and discussed in advance. Both during initial screening and at the beginning of each data collection session, study protocols and written consent forms were carefully explained so that participants understood data would be analyzed only when Mini-Cog scores were normal. No participant expressed concern about the results of the dementia test or asked about their scores.

Participants were willing to endure the somewhat tedious process of reading items aloud because they understood this permitted their peers to participate in the studies regardless of problems with vision, hearing or ability to read. All participants appeared to enjoy the sessions and many stated that they found the items on the questionnaires interesting or they were gratified to have their opinions sought and to be able to share their knowledge. An unexpected benefit to participants was that small group data collection sessions allowed for socializing with friends or meeting new acquaintances in open discussions and socialization after data collection was completed.

\section{RESULTS}

\section{Characteristics of the Sample}

Overall, the samples consisted of a diverse group of non-demented older adults aged 65 years and older. Characteristics of the samples - drawn from low-income, moderate income and 
upper-income CCRCs as well as from low-income and moderate-income senior centers-are displayed in Table 1.

\section{--- TABLE 1 HERE ---}

Average age of the sample as a whole was 79.5 years, with a range of $65-104$ years. As expected, mean age was appreciably greater for the two CCRC groups $(M=84)$ than for the community-dwelling groups $(M=76)$. Overall, $78 \%$ of participants were female, with a higher percentage of females among both of the low-income groups (86\%) compared to the upper- or moderate-income groups (71\%). Sixty-five percent of the total of all groups was White. At the low-income CCRC, $77 \%$ of participants were White and at the upper-income CCRC, $88 \%$ were White, allowing for some diversity in both settings. However, among the community-dwelling groups, race was sharply divided between low-income (100\% African American) and moderateincome groups (96\% White). On the whole, $70.5 \%$ of participants lived alone. A greater proportion of low-income participants (81\%) lived alone than moderate- or upper-income participants (60\%). For all participants, $45 \%$ of the total sample had a high school education or less, but only $18 \%$ of upper-income participants fell into this category. The proportion of participants in other groups with a high school education or less ranged from 38\% to 57\%.

\section{Collecting Data Among Small Groups}

Collecting survey data in small groups was contingent on strict adherence to the standardized protocol to assure independence of responses. When the rationale for maintaining silence and avoiding asking for items to be explained or rephrased was discussed, participants willingly complied, refraining from sharing their responses or influencing other's responses in any way. The physical setup of the space, with ample room between participants, was important 
also. The advantage of collecting data in small groups, among both random and convenience samples, was reflected in the brief time and limited use of study personnel necessary to complete data collection in all three studies, as illustrated in Table 2.

Data collection required only one researcher for each session and ranged from 6 days ( $N$ $=20)$ to 21 days $(N=123)$. When two researchers were available to schedule sessions simultaneously at separate sites, recruitment and data collection were completed $(N=152)$ in only 20 days. Accommodations for literacy and sensory deficits enabled all but one participant to successfully complete the data collection process.

\section{--- TABLE 2 HERE ---}

\section{Minimizing Exclusion Due to Dementia}

In the pilot study $(N=20)$, participants with dementia—as later identified by abnormal scores on the Mini-Cog test - were unable to comprehend instructions or complete the survey process without assistance. Some disruption of the group process occurred. In addition, because data from participants with abnormal Mini-Cog scores was excluded, but expenses were still incurred for recruitment and compensation, it was important to identify persons with cognitive deficits during initial telephone screenings and to minimize enrolling participants who subsequently were found to have abnormal scores.

In the two later studies, improved initial telephone screening by experienced gerontological nurses, using minimal reminders of scheduled sessions and avoiding follow-up for missed appointments were important mechanisms for reducing enrollment of participants for whom data was subsequently excluded because of abnormal dementia test scores, as demonstrated in Table 3. 


\section{--- TABLE 3 HERE ---}

The ability to combine screening and data collection in single sessions was contingent on limiting the number of participants with abnormal dementia scores. Exclusion of data from surveys because of abnormal Mini-Cog scores decreased significantly when reminders and follow-up were eliminated, as indicated by the difference between the first study, which used multiple reminders and phone calls to follow-up on missed sessions with $17.4 \%$ of participants excluded, and the second study in which the rate dropped to $6.5 \%$. For the third multisite study, the rate of exclusion because of dementia dropped even further to $3.3 \%$, at least partially related to the low incidence of dementia in the community-dwelling samples recruited at senior citizen centers.

\section{DISCUSSION}

Recruitment was conducted at multiple community sites, including CCRCs and senior citizen centers. These sites, although relatively homogeneous individually, together produced a diverse sample with a range of age, gender, race, marital status, education, and socioeconomic status representative of the older adult population. Data collection accommodated for persons with mild cognitive, sensory, or literacy deficits—an important segment of the older adult population which is often missed using other survey methods. Significant savings in time and costs were realized in all three studies.

Completion of written informed consent, dementia screening, and data collection in a single session produced a significant decrease in the amount of time needed for data collection, with a stratified random sample $(N=123)$ recruited and data collection completed in 3 weeks and a convenience sample $(N=152)$ in less than 4 weeks and only one investigator required per 
session. It was important to minimize the number of consented participants with subsequent abnormal dementia tests, both to prevent distress for vulnerable participants with cognitive impairment and to minimize costs (investigator time, compensation, printing, refreshments) incurred without obtaining usable data. Careful initial telephone screening and methods of scheduling with minimal reminders and follow-up accomplished this goal.

Findings in these studies were limited by their cross-sectional design and small sample sizes. Further study is necessary to see if these methods may be useful in other samples and settings.

\section{CONCLUSIONS}

Although recruitment and data collection among older adults can be challenging, several things have been learned in the three studies discussed in this article. First, it is feasible to conduct survey data collection in small groups while preserving scientific rigor as well as the privacy of participants. Second, it is feasible to limit recruitment of participants who lack the cognitive ability to complete the survey process and do not meet the inclusion criterion of a normal Mini-Cog score by using careful initial telephone screening, limiting reminders of scheduled sessions, and avoiding follow-up when sessions are missed.

Third, it is possible to compress written consent, dementia screening, and completion of questionnaires into single sessions while maintaining the rights of human subjects and abiding by a standardized protocol. And finally, participants are willing to take the extra time and effort to accommodate their peers with sensory or literacy limitations, resulting in samples more representative of the older adult population in which these limitations are common.

Significant savings in investigators' time and in costs for recruitment and data collection 
were realized using these methods. Given the scarcity of research funding and researchers' limited available time, other researchers may find it beneficial to consider one or more of these methods in future.

NOTE: A continuing care retirement community $(\mathrm{CCRC})$ is a residential complex that offers various levels of care for older adults such as independent and assisted living and skilled nursing. Residents of skilled nursing facilities or specialty units were not included in these studies. CCRCs included private apartments in which older adults resided either independently or receiving assisted living services such as minor help with personal care, accompanying a resident for shopping or medical appointments, or supervision of self-administered medications.

\section{REFERENCES}

Blackman, D. K., Kamimoto, L. A., \& Smith, S. M. (1999). Overview: Surveillance for selected public health indicators affecting older adults—United States. CDC Surveillance Summaries: Morbidity and Mortality Weekly Report, 48(8), 1-6.

Boles, M., Getchell, W. S., Feldman, G., McBride, R., Hart, R. G. (2000). Primary prevention studies and the healthy elderly: Evaluating barriers to recruitment. Journal of Community Health, 25(4), 279-292.

Borson, S., Scanlan, J., Brush, M., Vitaliano, P., \& Dokmak, A. (2000). The mini-cog: A cognitive "vital signs" measure for dementia screening in multi-lingual elderly. International Journal of Geriatric Psychiatry, 15(11), 1021-1027. Retrieved May 2, 2008, from http://geriatrics.uthscsa.edu/educational/med_students/minicog_htm

Centers for Disease Control and Prevention. (2009). Improving Health Literacy for Older Adults: Expert Panel Report 2009. Retrieved October 12, 2010, from http://www.cdc.gov/health 
marketing/healthliteracy/reports/olderadults.pdf

Folstein, M. F., Folstein, S. E., \& McHugh, P. R. (1975). "Mini-Mental State.” A practical method for grading the cognitive state of patients for the clinician. Journal of Psychiatric Research, 12(3), 189-198.

Katula, J. A., Kritchevsky, S. B., Guralnik, J. M., Glynn, N. W., Pruitt, L., Wallace, K., et al. (2007). Lifestyle interventions and independence for elders pilot study: Recruitment and baseline characteristics. Journal of the American Geriatrics Society, 55, 674-683.

Knäuper, B., Schwarz, N., Park, D., \& Fritsch, A. (2007). The perils of interpreting age differences in attitude reports: Question order effects decrease with age. Journal of Official Statistics, 23(4), 515-528. Retrieved December 16, 2010, from http://www.jos.nu/Articles/article.asp

McCarthy, V. L. (2009a, October). A feasibility study: Exploring the relationship of transcendence and adaptation with a new view of successful aging. Poster presented at the Research!Louisville Annual Research Conference in Louisville, Kentucky.

McCarthy, V. L. (2009b, November). Exploring a new theory of successful aging among lowincome older adults in a retirement community. Poster presented at the $62 \mathrm{nd}$ annual meeting of the Gerontological Society of America, Atlanta, Georgia.

McCarthy, V. L. (2011). Exploring a new theory of successful aging among low-income older adults in an independent and assisted living community. Journal of Theory Construction and Testing, 15(1), 17-21.

McDermott, M. M., Domanchuk, K., Dyer, A., Ades, P., Kibbe, M., \& Criqiu, M. H. (2009). Recruiting participants with peripheral arterial disease for clinical trials: Experience from the study to improve leg circulation (SILC). Journal of Vascular Surgery, 49(3), 653-59. 
Meiner, S. E., \& Lueckenotte, A. (2005). Gerontologic nursing (3rd ed.). Philadelphia, PA: Elsevier Health.

New England States Consortium Proceedings. (2000, December 7-8). Designing effective survey methods for frail elders: MMIP Technical Assistance Paper No. 11. A technical assistance paper of the Robert Wood Johnson Foundation Medicare/Medicaid Integration Program and the University of Maryland Center on Aging. Retrieved July 22, 2011, from http://www.gmu.edu/centers?chpre/research/MMIP/TApapers11.pdf

Ory, M. G., Lipman, P. D., Karlen, P. L., Gerety, M. B., Stevens, V. J., Singh, M. A., et al. (2002). Recruitment of older participants in frailty/injury prevention studies. Prevention Science, 3(1), 1-22.

Parker, C. J., \& Dewey, M. E. (2000). Assessing research outcomes by postal questionnaire with telephone follow-up. TOTAL Study Group. Trial Occupational Therapy and Leisure. International Journal of Epidemiology, 29(6), 1065-1069.

Sullivan-Bolyai, S., Bova, C., Deatrick, J. A., Knafl, K., Grey, M., Leung, K., et al. (2011). Barriers and strategies for recruiting study participants in clinical settings. The Gerontologist, 51(Suppl. 1), S33-S45.

U.S. Department of Health and Human Services. (2009). Research involving individuals with questionable capacity to consent: Points to consider. Office of Extramural Research, National Institutes of Health. Retrieved July 23, 2011, from http://grants.nih.gov/grants/policy/questionablecapacity.htm

Woodall, A., Morgan, C., Sloan, C., \& Howard, L. (2010). Barriers to participation in mental health research: Are there specific gender, ethnicity and age related barriers? $B M C$ Psychiatry, 10, 103-113. 


\section{Recruitment}

- For convenience samples (Studies 1 \& 3):

- Flyers and presentations were used by investigators; potential participants were invited to contact investigators by telephone for information about enrolling.

- Staff at sites approached potential participants and invited them to contact investigators by telephone for information.

○ Key informants, that is, participants who had completed data

\section{Initial Screening:}

Potential participants were told about the nature of the study and screened for inclusion criteria, using a standardized script, then scheduled for a data collection session at a time of their choice. Screening was done by the PI, an experienced gerontological nurse. Inclusion criteria were:

- self-reported, age 65 years or older;

- physically able to complete questionnaires in a small group setting at the CCRC or senior citizen center:

\section{Scheduling data collection:}

- Multiple data collection sessions (1 to -1.5 hours each) were available, with no more than eight participants scheduled per session.

- Participants selected a session at a date and time of their choice.

-Written reminders were placed in mailboxes at the CCRC; no reminders were provided at the community sites.

- Participants were given a phone number to call if they needed to cancel or reschedule.

Data collection:

- The nature of the study and inclusion criteria were explained.

-Written consent was signed.

- Questionnaire packets were completed, including:

- Mini-Cog Dementia Test (3-item recall and clock face drawing

- four standardized questionnaires (PCI, STS,. SAI, LSITA-SF),

o investigator-developed questionnaire for demographic data, and

- standardized questionnaire to assess functional status (Lawton IADL

Scale).

- Data from participants with abnormal Mini-Cog scores were excluded, with participants' consent.

- No follow-up was done if participants did not attend the scheduled data collection session because pilot study indicated that non-attendance was associated with abnormal Mini-Cog scores 
TABLE 1

\section{Characteristics of the Samples*}

\begin{tabular}{|c|c|c|c|}
\hline CCRC & Low-income & Upper-Income & Total \\
\hline Age in years & $\mu=80(65-104)$ & $\mu=87(74-102)$ & $\mu=84(65-104)$ \\
\hline Female & $80 \%$ & $69 \%$ & $74 \%$ \\
\hline$\%$ White & $77 \%$ & $88 \%$ & $82 \%$ \\
\hline Lives alone & $90 \%$ & $60 \%$ & $75 \%$ \\
\hline High school or less & $57 \%$ & $18 \%$ & $38 \%$ \\
\hline Community & Low-income & Moderate & Total \\
\hline Age & $\mu=76(65-94)$ & $\mu=74(65-97)$ & $\mu=75(65-97)$ \\
\hline Female & $92 \%$ & $73 \%$ & $82 \%$ \\
\hline$\%$ White & $0 \%$ & $96 \%$ & $48 \%$ \\
\hline Lives alone & $72 \%$ & $59 \%$ & $66 \%$ \\
\hline High school or less & $51 \%$ & $54 \%$ & $52 \%$ \\
\hline
\end{tabular}


TABLE 2

\begin{tabular}{|c|c|c|c|c|c|c|}
\hline Study & Site(s) & $\begin{array}{l}\text { Type of } \\
\text { sample }\end{array}$ & $\begin{array}{l}\text { Sample } \\
\text { size }\end{array}$ & $\begin{array}{l}\text { \# of days } \\
\text { to complete }\end{array}$ & $\begin{array}{c}\text { \# of } \\
\text { sessions }\end{array}$ & $\begin{array}{c}\text { \# of } \\
\text { researchers }\end{array}$ \\
\hline 1 & $\begin{array}{l}\text { Moderate- } \\
\text { income } \\
\text { CCRC }\end{array}$ & Convenience & $N=20$ & 6 & 3 - 4/day & 1 \\
\hline 2 & $\begin{array}{l}\text { Low-income } \\
\text { CCRC }\end{array}$ & $\begin{array}{l}\text { Random, } \\
\text { stratified by } \\
\text { independent } \\
\text { or assisted } \\
\text { living status }\end{array}$ & $N=123$ & 21 & 3 - 4/day & 1 \\
\hline 3 & Multi site: & Convenience & $N=152$ & 19 & 26 & 2 \\
\hline & $\begin{array}{l}\text { a) Upper- } \\
\text { income } \\
\text { CCRC }\end{array}$ & & $n=51$ & 5 & 11 & 1 \\
\hline & $\begin{array}{l}\text { b) Low- } \\
\text { income } \\
\text { senior } \\
\text { center }\end{array}$ & & $n=49$ & & 8 & 1 \\
\hline & c) & & & 8 & & \\
\hline & $\begin{array}{l}\text { Moderate- } \\
\text { income } \\
\text { senior } \\
\text { center }\end{array}$ & & $\mathrm{n}=52$ & & 7 & 1 \\
\hline
\end{tabular}


INNOVATIVE RESEARCH METHODS

TABLE 3

Exclusion Related to Dementia

Study/Sample

\# Recruited

Sample size

\# Excluded

Percent

excluded

1. Moderate-

24

20

4

$17.4 \%$

income

CCRC

2. Low-income

131

123

8

$6.5 \%$

CCRC

3. Multi-site

157

152

5

$3.3 \%$ 